\title{
Irreversible electroporation-mediated shRNA knockdown of the HPV18 E6 gene suppresses cervical cancer growth in vitro and in vivo
}

\author{
ZHI-LIANG WANG ${ }^{1}$, WEI ZHOU ${ }^{2}$, ZHENG-AI XIONG $^{1}$, TENG-HUA YU ${ }^{3}$, LI-MEI WU ${ }^{1}$, CHENG-XIANG LI $^{4}$, \\ CHENG-GUO YAO ${ }^{4}$, YU-TONG WU ${ }^{1}$ and YUAN-YUAN HUA ${ }^{1}$ \\ ${ }^{1}$ Department of Obstetrics and Gynecology, The Second Affiliated Hospital of Chongqing Medical University, \\ Chongqing 400010; ${ }^{2}$ Department of Obstetrics, Chongqing Health Center for Women and Children, Chongqing 400013; \\ ${ }^{3}$ Department of Breast Surgery, Jiangxi Cancer Hospital, Nanchang 330029; ${ }^{4}$ State Key Laboratory of \\ Power Transmission Equipment and System Security and New Technology, \\ Chongqing University, Chongqing 400044, P.R. China
}

Received July 29, 2015; Accepted January 12, 2017

DOI: $10.3892 / 01.2017 .6405$

\begin{abstract}
Irreversible electroporation (IRE) is a physical, non-thermal cancer therapy, which leads to cell death via permanent membrane permeability. This differs from reversible electroporation (RE), which is used to transfer macromolecules into target cells via transient membrane permeability. Given the electrical impedance of the electric field, RE co-exists outside the central zone of IRE ablation. In the present study, the feasibility of using IRE at a therapeutic dose to mediate short hairpin RNA (shRNA) knockdown of human papillomavirus (HPV)18 E6 in HeLa cervical cancer cells in vitro and in vivo was investigated. Experimental results indicated that the HeLa cells survived the combined treatment with IRE and shRNA plasmid transfection. Additionally, residual tumor tissue in a nude mouse model demonstrated green fluorescence. Subsequent studies showed that the combined treatment inhibited the growth of HeLa cells and tumors. Western blotting analysis showed marked changes in the growth-associated proteins between the combined treatment group and the control. It was concluded that a therapeutic dose of IRE was able to mediate the transfection of HPV18 E6 shRNA into HeLa cervical cancer cells in vitro and in vivo. This combined treatment strategy has promising implications
\end{abstract}

Correspondence to: Professor Zheng-Ai Xiong, Department of Obstetrics and Gynecology, The Second Affiliated Hospital of Chongqing Medical University, 76 Lin Jiang Road, Yuzhong, Chongqing 400010, P.R. China

E-mail:docxza@163.com

Key words: irreversible electroporation, human papillomavirus, interference plasmid, cervical cancer, tumor growth, xenograft model in cancer treatment for the ablation of tumors, and in eliminating microscopic residual tumor tissue.

\section{Introduction}

Electroporation is a promising, minimally invasive technique that is able to increase the permeability of cell membranes and tissues located in the externally applied pulsed electric fields (1). The consequence of permeability varies as increasing voltage is applied. Reversible electroporation (RE) occurs under a relatively low voltage and causes the permeability of the cell membrane to increase temporarily, meaning the treated cells survive (2). By contrast, irreversible electroporation (IRE) happens when the pulsed electric fields exceed a certain threshold and the treated cells are killed (3).

Neumann et al (1) have demonstrated a medical application of electroporation by using pulsed electric fields to temporarily permeabilize cell membranes and deliver foreign DNA into cells. Strategies of employing a combination of pulsed electric fields and chemotherapeutic drugs or DNA (small molecules compared to usual plasmid sizes) emerged in the following decades, namely electrochemotherapy (ECT) (4) and gene electrotransfer (5). RE has been mainly used in combination strategies to temporarily increase permeability, whilst keeping the tissues and cells alive so the transfected small molecules (e.g., chemotherapeutic drugs, short hairpin RNA (shRNA) vector, or DNA vaccine) can bring about therapeutic benefits $(6,7)$.

Until 2005, Davalos et al (8) proposed the term IRE to distinguish between cell destruction and RE by using electroporation as a monotherapy without employing any small molecules to destroy tissues. Subsequently in 2010 , Pech et al (9) first reported a human clinical study (kidney tumor; $n=6$ ) where electroporation was applied as a means of soft tissue destruction. A number of pre-clinical tests have been reported in various other types of tumor including liver $(10,11)$, lung (8), pancreatic (12) and prostate (13). Therefore, IRE has been considered as a novel, physical cancer treatment. 
However, it is notable that the tissue heterogeneity in structure affects electric conductivity and electric field distribution, and thus cell survival upon IRE treatment, due to the 'electric field sinks' effect (14), and the volume of a single time ablation is $<1 \mathrm{~cm}^{3}$ without repositioning the electrodes $(15,16)$.

In a previous study, Joyce et al (17) hypothesized that outside the central zone of IRE ablation exists a peripheral zone of reversible electroporation, where gene transfer may occur. This was demonstrated by performing IRE in the liver of a Yorkshire pig model, and by administrating a green fluorescent protein (GFP)-labeled plasmid by bolus or primed infusion through the hepatic artery or portal vein. It is notable that this study used a high concentration of plasmid, delivered through the blood vessels and the study was conducted in the liver of a healthy pig model (17). Therefore, in the present study, the feasibility of using IRE to mediate human papillomavirus (HPV)18 E6 shRNA plasmid transfection into cervical cancer cells in vitro and in vivo was investigated, and the effect of this combined treatment on tumor growth was observed.

\section{Materials and methods}

shRNA plasmids. The enhanced (E)GFP labeled pGenesil-1 plasmid (Shanghai GeneChem Co., Ltd., Shanghai, China) was used to construct the shRNA plasmid targeting the HPV18 E6 gene, as previously described (18). shRNA targeted the HPV18 E6 coding region at nucleotides 391-411 in intron 1 of the HPV18 bicistronic transcripts. A total of two pairs of DNA oligonucleotides (Beijing Dingguo Changsheng Biotechnology Co., Ltd., Beijing, China) were cloned into the BamHI/HindIII restriction site of the pGenesil-1 plasmid. The sequences for the sense and antisense strands were 5'-GATCCCTGGGTT ATACAATTTATTAATTCAAGAGATTAATAAATTGTA TAACCCAGTGA-3' and 3'-GGACCCAATATGTTAAATAA TTAAGTTCT CTAATTATTTAACATATTGGGTCACTT CGA-5', respectively. The negative control shRNA (Beijing Dingguo Changsheng Biotechnology Co., Ltd.) had limited homology to any known sequences in the human genome. The sequences for the sense and antisense strands were 5'-GATCCGGAGTACCCTGATG AGATCTT CAAGAGAGA TCTCATCAGGGTACTCCTGA-3' and 3'-GCCTCATGGG ACTACTCTAGAAGTTCTCTCTAGAGTAGTCCCATGAG GACTTCGA-5', respectively. The constructed plasmids were amplified by polymerase chain reaction (PCR) and verified by DNA sequencing with forward primer (5'-AGGCGATTAAGT TGGGTA-3') and reverse primer (5'-CGGTAGGCGTGTACG GTG-3'). PCR amplification was performed using $3 \mu \mathrm{l} 10 \mathrm{X}$ rTaq polymerase buffer (Dingguo Changsheng Biotechnology Co., Ltd, Beijing, China), dNTP $2 \mu 1$, forward primer $1 \mu 1$, reverse primer $1 \mu \mathrm{l}$, DNA $2 \mu 1,0.2 \mu \mathrm{l}$ rTaq polymerase (Dingguo Changsheng Biotechnology Co., Ltd.), and $\mathrm{H}_{2} \mathrm{O} 20.8 \mu \mathrm{l}$. PCR amplification conditions included 30 cycles, each at $95^{\circ} \mathrm{C}$ for $5 \mathrm{~min}, 95^{\circ} \mathrm{C}$ for $30 \mathrm{sec}, 55^{\circ} \mathrm{C}$ for $30 \mathrm{sec}, 72^{\circ} \mathrm{C}$ for $45 \mathrm{sec}$ and $72^{\circ} \mathrm{C}$ for $5 \mathrm{~min}$. The PCR kits and reagents were purchased from Beijing Dingguo Changsheng Biotechnology Co., Ltd. The constructed positive control is named as E6 shRNA plasmid and the negative control named as CTL shRNA plasmid.

To verify the presence and function of the plasmid, cells were observed for expression of GFP under fluorescence microscopy following IRE treatment or transfection with Lipofectamine 2000 (Invitrogen; Thermo Fisher Scientific, Inc., Waltham, MA, USA).

Cell culture. HPV18-positive HeLa cervical carcinoma cells (Shanghai Cell Bank, Type Culture Collection Committee, Chinese Academy of Sciences, Shanghai, China) were maintained in Dulbecco's modified Eagle's medium (DMEM) supplemented with 10\% fetal bovine serum (FBS) (both from Hyclone; GE Healthcare Life Sciences, Logan, UT, USA) at $37^{\circ} \mathrm{C}$ in a $5 \% \mathrm{CO}_{2}$ humidified incubator.

Treatment of cells with IRE and plasmid transfection. Exponentially growing HeLa cells were collected and resuspended in RPMI-1640 (Gibco; Thermo Fisher Scientific, Inc., Waltham, MA, USA) (without FBS), with a final concentration of $2 \times 10^{6} / \mathrm{ml}$. Cells were divided into 6 groups and subjected to the following treatments: Group A, CTL (untreated control); group B, IRE; group C, transfected with $6 \mu \mathrm{g}$ CTL shRNA plasmid; group D, IRE + CTL shRNA; group E, E6 shRNA plasmid; and group F, IRE + E6 shRNA. Groups D and F were subjected to IRE treatment after $10 \mu \mathrm{g}$ of the appropriate plasmids were added in cell suspension, as described previously (19). Briefly, IRE was performed on each $500 \mu \mathrm{l}$ aliquot of HeLa cell suspension. Samples were placed in a parallel aluminum plated Gene Pulser Cuvette (Bio-Rad Laboratories, Inc., Hercules, CA, USA) with an electric pulses therapeutic system (State Key Laboratory of Power Transmission Equipment and System Security and New Technology, Chongqing University, Chongqing, China) at a pulse parameter of $1 \mathrm{~Hz}$ and $800 \mathrm{~V}$, and for 10 pulses at a duration of $100 \mu$ s for each pulse. Groups $\mathrm{C}$ and $\mathrm{E}$ were transfected with $6 \mu \mathrm{g}$ of the appropriate plasmid using Lipofectamine 2000 reagent in OPTI-MEM medium (Invitrogen, Thermo Fisher Scientific, Inc.) according to the manufacturer's instructions for $6 \mathrm{~h}$. Group B was treated with IRE alone.

Total RNA isolation and reverse transcription-quantitative PCR (RT-qPCR). Total RNA was isolated from cultured HeLa cells using the RNAiso kit (Takara Biotechnology Co., Ltd., Dalian, China) according to the manufacturer's protocol. HPV18 E6 transcripts were detected using primers 5'-AGGCG ATTAAGTTGGGTA-3' and 5'-CGGTAGGCGTGTACG GTG-3'. The housekeeping gene GAPDH was used as a reference gene for normalization. Gene expression relative to GAPDH was determined using the $2^{-\Delta \Delta C q}$ method (20). qPCR was performed using a 2X Brilliant SYBR-Green QPCR Master Mix (Stratagene; Agilent Technologies, Inc., Santa Clara, CA, USA) as described previously (21). The qPCR cycling conditions included pre-incubation for $5 \mathrm{~min}$ at $94^{\circ} \mathrm{C}$, followed by 30 cycles of denaturation for $30 \mathrm{sec}$ at $94^{\circ} \mathrm{C}$ and annealing for $30 \mathrm{sec}$ at $50^{\circ} \mathrm{C}$, prior to an extension step for $30 \mathrm{sec}$ at $72^{\circ} \mathrm{C}$ and a final extension step for $10 \mathrm{~min}$ at $72^{\circ} \mathrm{C}$. PCR products were resolved and analyzed on $1 \%$ agarose gels containing $0.5 \%$ ethidium bromide (Beyotime Institute of Biotechnology, Haimen, China).

Western blotting. Protein extracts were prepared $48 \mathrm{~h}$ following transfection or IRE treatment and subsequently subjected to western blot analysis for HPV18 E6, p53 and proliferating 
cell nuclear antigen (PCNA). A total of $50 \mu \mathrm{g}$ protein was loaded into each lane and separated by SDS-PAGE $(12.5 \%$ gel), transferred to polyvinylidene difluoride membranes, and immunoblotted with primary antibodies followed by incubation with a goat anti-mouse-HRP conjugated secondary antibody (dilution, 1:5,000; BIOSS, Beijing, China). Finally, detection procedures were performed using Immobilon Western Chemiluminescent HRP substrate (EMD Millipore, Billerica, MA, USA). Primary antibodies against the following proteins were used: HPV18 E6 (catalog no. sc-460; dilution, 1:500), p53 (catalog no. sc-47698; dilution, 1:1,000), PCNA (catalog no. sc-25280; dilution, 1:1,000) (all from Santa Cruz Biotechnology, Inc., Dallas, TX, USA) and GAPDH (catalog no. bs-10900R; dilution, 1:1,000; BIOSS). Proteins were visualized with enhanced chemiluminescent reagent (ECL; Thermo Fisher Scientific, Inc.) and bands quantified using Quantity One software (version 4.4; Bio-Rad Laboratories, Inc.).

Cell proliferation by cell-counting kit-8 (CCK-8 assay). To assay the growth of HeLa cells, cells suspended in DMEM $\left(100 \mu \mathrm{l}\right.$ of $2 \times 10^{4}$ cells $\left./ \mathrm{ml}\right)$ were seeded into each well of 96-well culture plates and cultured for 24, 48, 72 and $96 \mathrm{~h}$ at $37^{\circ} \mathrm{C}$ (in a humidified incubator with a $5 \% \mathrm{CO}_{2}$ atmosphere). Subsequently, $10 \mu \mathrm{l}$ of CCK-8 solution (Dojindo Molecular Technologies, Inc., Kumamoto, Japan) dissolved in DMEM was added to each well, followed by incubation at $37^{\circ} \mathrm{C}$ for $2 \mathrm{~h}$. The number of viable cells was assessed by measurement of absorbance at $450 \mathrm{~nm}$ using a microplate reader (Bio-Rad Laboratories, Inc.). The relative cell number of each group was calculated as OD value at different time points/OD value when the cells just adhere to the wall ( $\sim 6 \mathrm{~h}$ following seeding). All samples were tested in triplicate, and the differences between the controls and the test groups were analyzed.

Animal experiments. Ethical approval of animal care and experiments was obtained from the Second Affiliated Hospital of Chongqing Medical University (Chongqing, China). Specific pathogen-free athymic (T-cell deficient) nude mice (BALB/c nude; 4-6 weeks old; female) were obtained from the Animal Experiment Center of Chongqing Medical University (Chongqing, China). Suspensions of $2 \times 10^{6}$ cells in $0.2 \mathrm{ml} \mathrm{PBS}$ were injected into the dorsal subcutis of the mice $(n=21)$ by sterile syringes. In order to verify that plasmids could be transfected by irreversible electroporation and expressed in vivo when the diameter of the subcutaneous tumors reached 8-10 $\mathrm{mm}$ ( 30 days following subcutaneous injection of HeLa cells suspension), one tumor was collected as a pre-test. IRE was performed within 10 min after $10 \mu \mathrm{g}$ E6 shRNA plasmid was injected into the tumor tissue at multiple points. The tumor was harvested to perform histopathology and fluorescence microscopy using frozen sections 48 h later. Subsequently, the remaining mice $(n=20)$ were randomly divided into four groups: i) group 1 (control), received no treatment; ii) group 2 , received only IRE (800 V; $100 \mu \mathrm{s} ; 1 \mathrm{~Hz} ; 10$ pulses); iii) group 3, received intratumoral injection of $10 \mu \mathrm{g}$ isolated plasmid alone at multiple points; iv) group 4, received both treatments (IRE was performed within $10 \mathrm{~min}$ after $10 \mu \mathrm{g}$ plasmid was injected into the tumor tissue at multiple points). The tumors were measured every seven days with a caliper until the animals were sacrificed by the cervical dislocation method at day 28 following subcutaneous injection of HeLa cells suspension, since a subcutaneous transplant tumor with growth of $>1$ month or that is $>1 \mathrm{~cm}$ in diameter is prone to necrosis. Tumor volume was calculated by the following formula: $\mathrm{V}=\pi a b c / 6$, where $\mathrm{V}$ is the volume, $a$ is the maximum diameter, and $b$ and $c$ are the other two perpendicular diameters.

Histology and microscopy. Initially, the tumor that received combined treatment was harvested and cut in half to identify the IRE ablation effect, the feasibility of IRE-mediated plasmid transfer into tumor tissue and the expression of plasmid. One half of the specimen was fixed in $10 \%$ neutral buffered formalin for histopathology, and the other half was freshly frozen in optimal cutting temperature compound (Tissue-Tek; Sakura Finetek USA, Inc., Torrance, CA, USA) for fluorescence microscopy. Formalin-fixed tissues were processed routinely, sectioned at $4 \mu \mathrm{m}$ thickness and stained with hematoxylin and eosin. Frozen tissues were cut into $10 \mu \mathrm{m}$ sections and stained with DAPI, and observed under a fluorescence microscope (Nikon Eclipse TE300; Nikon Corporation, Tokyo, Japan) equipped with a GFP emission filter to detect green fluorescence as well as a TRIT-C filter to detect autofluorescence. Images were acquired on NIS-Elements Basic Research software (version 2.30; Nikon Corporation, Tokyo, Japan).

Statistical analysis. All experiments were performed in triplicate. $\mathrm{P}<0.05$ was considered to indicate a statistically significant difference. Results were statistically analyzed with one-way analysis of variance (ANOVA) and Post-hoc ANOVA Tukey's HSD test or unpaired t-test at 5\% level of significance. Statistical analysis was performed using GraphPad Prism software (version 5.0; GraphPad Software, Inc., La Jolla, CA, USA).

\section{Results}

IRE induces the transfection of E6 shRNA plasmid into HeLa cells and results in E6 $m R N A$ knockdown. An EGFP labeled HPV18 E6 shRNA plasmid was successfully constructed and identified by DNA sequencing. To verify the feasibility of using IRE to transduce a plasmid into HeLa cells, the expression of GFP was observed $48 \mathrm{~h}$ after IRE $(800 \mathrm{~V}$; $100 \mu \mathrm{s} ; 1 \mathrm{~Hz}$; 8 pulses) treatment under an inverted fluorescence microscope. Few HeLa cells survived $24 \mathrm{~h}$ after IRE combined with plasmid treatment. Strong green fluorescence was observed in the cells that survived (Fig. 1A-D).

HPV18 E6 mRNA expression was then measured by qPCR. The knockdown efficiency of HPV18 E6 mRNA level was up to $90 \%$. There were statistically significant differences in HPV18 E6 mRNA expression between CTL group and IRE+E6 shRNA group $(\mathrm{P}<0.01$; Fig. $1 \mathrm{E})$, the difference also appeared between IRE + CTL shRNA group and IRE+E6 shRNA group $(\mathrm{P}<0.05$, Fig. 1E).

Combination of HPV18 E6 shRNA plasmid transfection and IRE inhibits HeLa cell proliferation in vitro. A previous study demonstrated that tumor cell proliferation is positively dependent on E6 levels and HPV DNA load (21). The present study confirmed that IRE induced the transfection of interference 


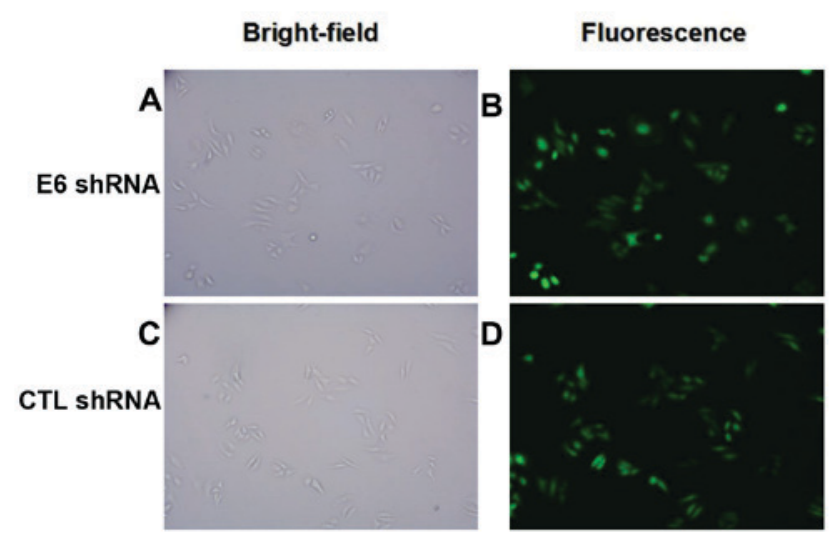

E

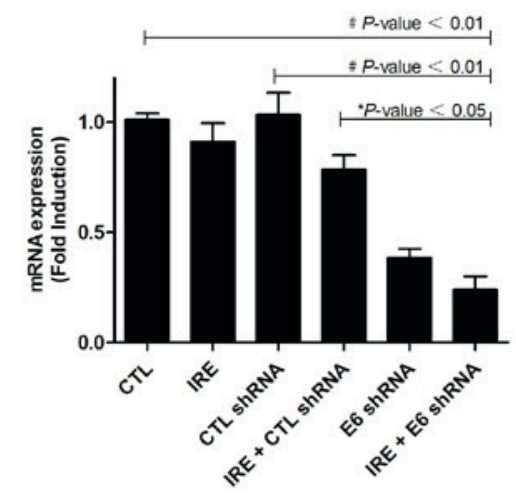

Figure 1. IRE induces plasmid transfer into HeLa cells successfully. Representative images of GFP expression $48 \mathrm{~h}$ post-combination treatment of HeLa cells with (A and B) IRE and E6 shRNA and (C and D) CTL shRNA plasmid. The results revealed no significant difference in the rate of GFP-positive cells (magnification, x200) between the nonspecific plasmic and the E6 targeted shRNA plasmid. (E) The co-effect of the plasmid and IRE on HPV18 E6 mRNA level was investigated by quantitative polymerase chain reaction $48 \mathrm{~h}$ post-treatment, and GAPDH served as the housekeeping gene. The results are depicted as the mean \pm standard deviation for at least three separate experiments. ${ }^{*} \mathrm{P}<0.05,{ }^{~} \mathrm{P}<0.01$. CTL, control; GAPDH, glyceraldehyde-3-phosphate dehydrogenase; GFP, green fluorescent protein; HPV18 E6, human papillomavirus type 18 E6 protein; IRE, irreversible electroporation; shRNA, short hairpin RNA.

plasmid into HeLa cells, and the proliferation of which was inhibited; however, there were two interfering factors (plasmid and IRE), so one factor may have worked independently. CCK-8 assay was performed to investigate the proliferation of HeLa cells. The growth curves $(\mathrm{P}<0.01$; Fig. $2 \mathrm{~A})$ of the six groups showed that the combination treatment with IRE and E6 shRNA significantly inhibited HeLa cell proliferation vs. the CTL group, and lower than the E6 shRNA plasmid group (pGenesil-E transfected by Lipofectamine 2000) for $96 \mathrm{~h}$, although this difference was not statistically significant. Furthermore, western blotting was performed to compare the relative protein level among the six groups. The western blot showed similar results to the CCK- 8 curve. HPV18 E6 oncoprotein was significantly downregulated in pGenesil-E transfected groups: E6 shRNA plasmid and IRE+E6 shRNA vs. the CTL group ( $\mathrm{P}<0.01$; Fig. $2 \mathrm{~B}$ and $\mathrm{C})$. The level of E6 downstream proliferation-associated proteins, including p53 and PCNA; p53 was upregulated while PCNA was downregulated. When E6 was downregulated in E6 shRNA plasmid and IRE+E6 ShRNA groups, p53 was upregulated and PCNA was downregulated. Therefore, the proliferation of these groups was lower than the control. Notably, there was a small non-significant difference between the E6 shRNA plasmid and IRE+E6 shRNA groups.

IRE induces plasmid transfection into tumor tissue and suppresses tumor growth in cervical xenograft model. Histopathological results demonstrated that ablated zones were well demarcated and part of the tumor remained intact under the fixed IRE parameter ( $800 \mathrm{~V} ; 100 \mu \mathrm{s} ; 1 \mathrm{~Hz} ; 10$ pulses). Necrosis, cytoplasmic hypereosinophilia, nuclear pyknosis and karyorrhexis can be observed in the ablated areas (Fig. 3A-C). Fluorescence microscopy revealed that plasmid transfection was induced by IRE into the residual tumor cell and the plasmids were expressed (Fig. 3D-F).

A total of 8 days following treatment, the tumor size was significantly reduced in IRE+E6 shRNA group compared with those in either the control group or single treatment (IRE or plasmid) groups $(\mathrm{P}<0.05$; Fig. $4 \mathrm{~A}$ and $\mathrm{B})$. To investigate the effect of the combined treatment with IRE and shRNA plasmid transfection, the tumors were harvested and western blotting was performed. The HPV18 E6 oncoprotein level was significantly decreased in the IRE + plasmid group. vs. the control group $(\mathrm{P}<0.05$; Fig. $4 \mathrm{C}$ and $\mathrm{D})$. An opposite trend was observed in the level of p53 protein, which is consistent with the mechanism of p53 degradation by E6 (22).

\section{Discussion}

IRE is a type of physical therapy for cancer based on electrical and biological effects of pulsed electric fields. The electric biological effects of electric pulse are known. In the 1970s, cell electroporation or electropermeabilization (defined as the permeabilization of the cell membrane induced by exposure to short and intense electric pulses) were used to increase the cellular uptake of normally non-permeable molecules (e.g., drugs, dyes or DNA) (23). Recently, electroporation-based treatments (based mainly on RE), including ECT and electrogenetherapy (EGT) have been employed in clinical settings (24). By modulating the electric pulses and the parameters of electroporation, permanent permeabilization may be observed under transmembrane potential which eventually leads to cell death; this is termed IRE. IRE has been used in the food industry for sterilization for decades (25) until in 2005 Davalos et al (8) proposed the concept of using electroporation as a monotherapy which is distinct from ECT or EGT.

IRE and RE co-exist and cannot be separated due to the existence of dielectric impedance (24). Given the inherent dielectric properties of the cell suspension or tumor tissue, the electric field strength decreases as the distance from needle electrode increases (24). A simplified example of this co-existence of IRE and RE is a shooting target of concentric circles with IRE in the middle and RE in the periphery.

IRE was considered as a major side effect in RE-based techniques (such as ECT or EGT). The cells in the target area survived following RE exposure. Electric field parameters were strictly controlled to avoid IRE, which could lead cell to death. Conversely, during the treatment of IRE, appropriate electric field parameters were selected to ensure that the target area was completely covered by IRE. The target area 

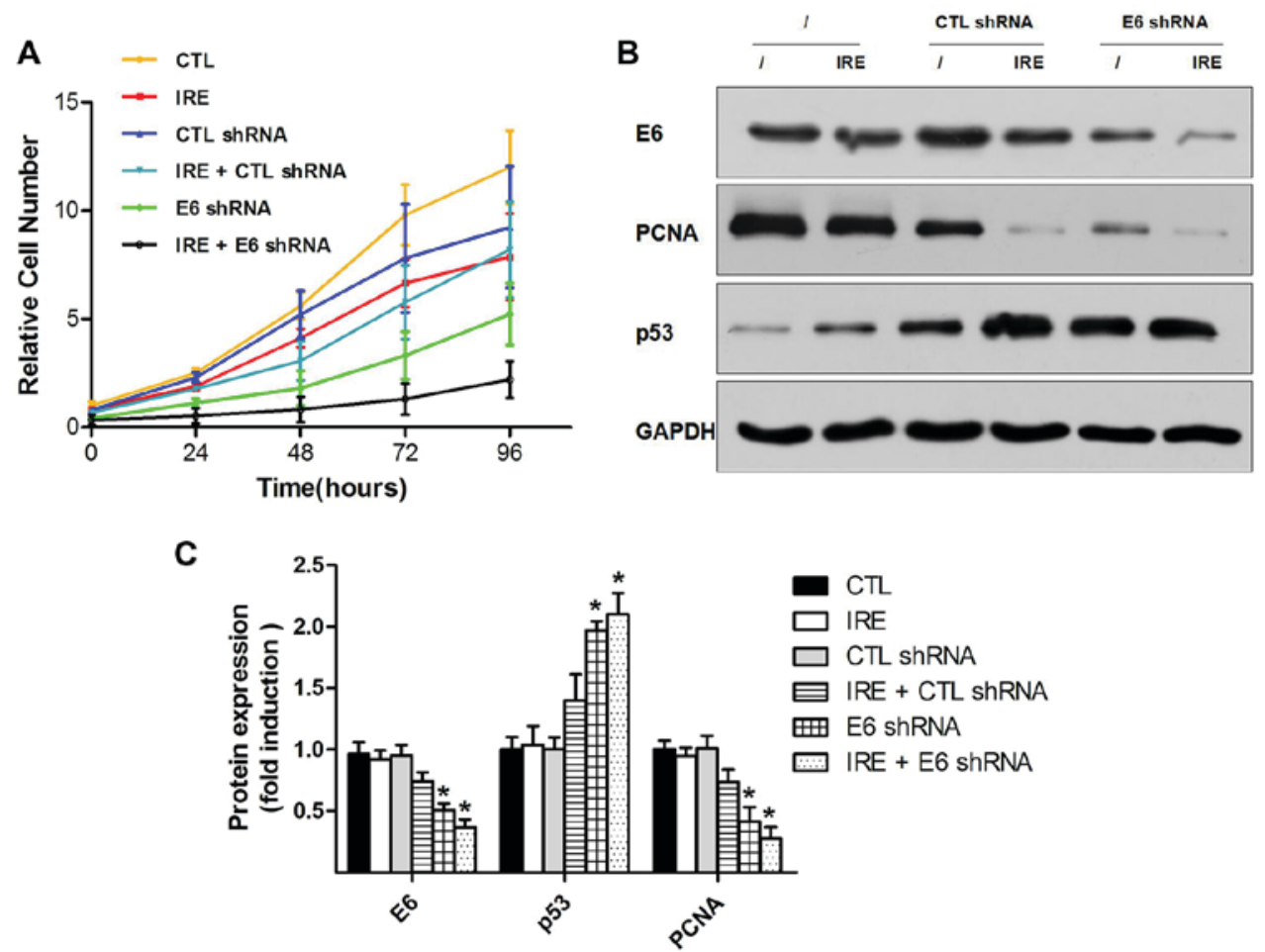

Figure 2. Effect of IRE and E6-shRNA plasmid transfection on HeLa cell growth. (A) Cell proliferation was assessed by the cell-counting kit-8 method and represented by relative cell number. (B and C) The protein levels of HPV18 E6, PCNA and p53 were examined by western blotting, and GAPDH served as the internal reference. Data are depicted as the mean \pm standard deviation from at least three separate experiments. " $\mathrm{P}<0.05$, vs. the control. CTL, control; PCNA, proliferating cell nuclear antigen; GAPDH, glyceraldehyde-3-phosphate dehydrogenase; HPV18 E6, human papillomavirus type 18 E6 protein; IRE, irreversible electroporation; shRNA, short hairpin RNA.

A
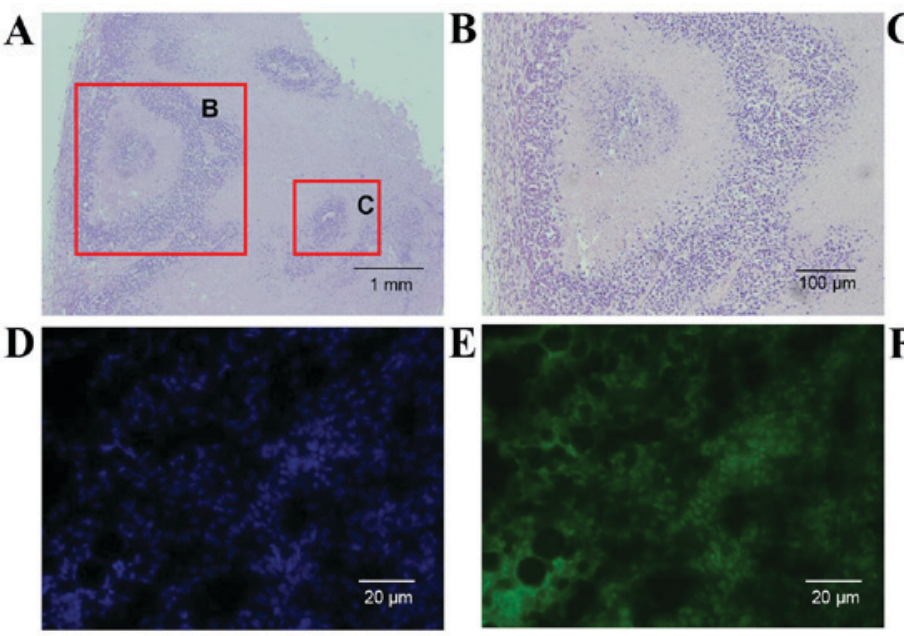
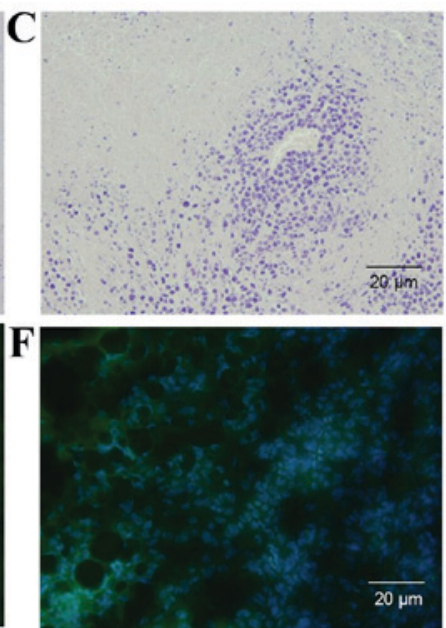

Figure 3. Incomplete ablation of IRE and the expression of GFP in the surviving tumor tissue. (A-C) Haematoxylin and eosin staining of representative IRE ablated area from one half of the tumor showed incomplete ablation as expected. Frozen sections of tumor tissues were obtained from the other half of the tumor and stained by DAPI, then observed by a fluorescence microscope. (D) DAPI staining. (E) GFP expression. (F) Merged images. GFP, green fluorescent protein; IRE, irreversible electroporation.

was destroyed by IRE. During IRE treatment, all RE activity in which reversible nanopores in the surface of the cells are formed, and the treated cells are not killed, needs to be minimized. In a Yorkshire pig model, Joyce et al (17) demonstrated that a peripheral zone of reversible electroporation, where gene transfer can occur, exists outside the central zone of IRE ablation. IRE was performed in the liver of a Yorkshire pig model with the administration of $7 \mathrm{mg}$ GFP-labeled plasmid via bolus or primed infusion directly through the hepatic artery or portal vein. This study showed that liver ablation by IRE was clearly demarcated on histology, and 31/36 liver specimens treated with IRE and the GFP plasmids demonstrated strong green fluorescence (17). In subsequent studies, it was observed that IRE facilitated gene transfer of the granulocyte-macrophage colony-stimulating factor plasmid and brought about a local and systemic biologic response (26). This demonstrated that 

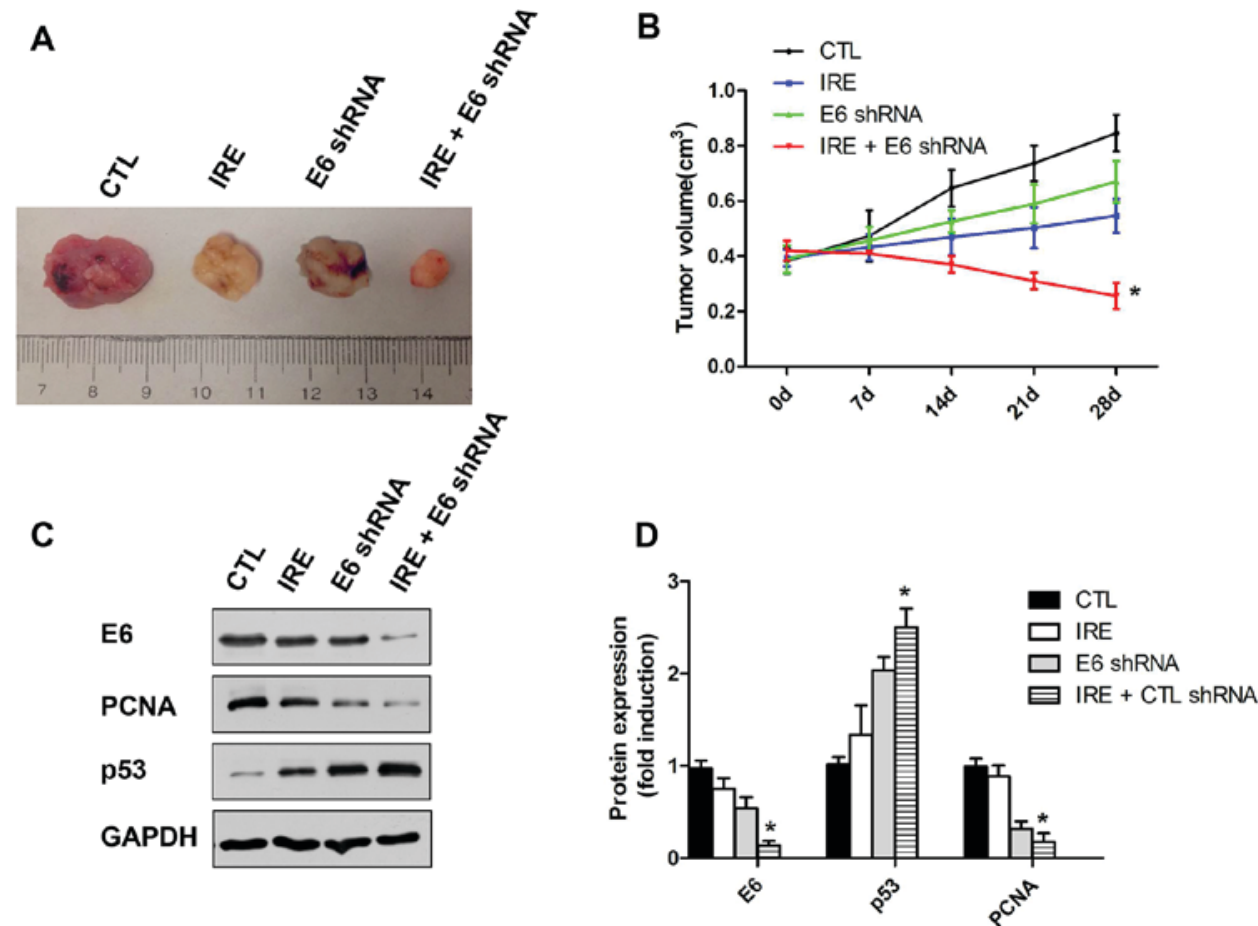

Figure 4. Co-effect of IRE and E6-shRNA plasmid on tumor growth. (A) Representative image of harvested tumors on day 30 post-treatment. (B) Tumor growth curve. (C and D) Western blotting analysis of proteins in the tumor tissues 30 days post-treatment. Data are depicted as the mean \pm standard deviation from at least three separate experiments. * $\mathrm{P}<0.05$, vs. the control. CTL, control; HPV 18 E6, human papillomavirus type 18 E6 protein; GAPDH, glyceraldehyde-3-phosphate dehydrogenase; IRE, irreversible electroporation; shRNA, short hairpin RNA; PCNA, proliferating cell nuclear antigen.

the technique holds the potential for tumor eradication and immunotherapy of residual cancer.

In the present study, by modulating the electric pulse parameters of IRE, a situation of incomplete ablation was simulated within a certain area (in a cuvette or a tumor). A therapeutic dosage [ $\geq$ IRE threshold of $667 \mathrm{~V} / \mathrm{cm}$ (27)] of pulsed field was projected to the subjects (cells or tumors), in which the co-existence of IRE and RE following treatment was observable. IRE treatment killed the majority of the HeLa cells in the cell suspension and ablated part of the tumors. The cells that survived showed green fluorescence under an inverted fluorescence microscope. Frozen sections of the treated tumor showed that the peripheral margin was intact and demonstrated strong green fluorescence. These results indicated a therapeutic dose of IRE was able to mediate plasmid transfection into the tumor in vivo and in vitro. Further results confirmed that the plasmid was expressed in the surviving tumor cells, and the effect of the combined treatment with IRE and shRNA was greater than the single treatment with IRE or shRNA. Notably, the resultant changes in protein levels and cell growth were more significant in the combined IRE and shRNA treatment group compared to the changes in the E6 shRNA transfected group, although no statistically significant difference was observed. These results may be due to a co-effect of IRE and shRNA plasmid transfection on tumor cells. This notable observation may be explored in future studies.

In conclusion, the present study verified the feasibility of utilizing IRE to mediate HPV-18 E6 shRNA transfection into cervical cancer HeLa cells in vitro and in vivo. The shRNA plasmid was well expressed in HeLa cells in vitro and in vivo, and the interference effect was detected by PCR, western blotting and CCK- 8 assay. This combined treatment strategy has promising implications in cancer treatment for the ablation of tumors and in eliminating microscopic residual tumor tissue.

\section{Acknowledgements}

The present study was supported by the National Natural Science Foundation of China (grant nos. 81201745 and 81301928), the Health and Family Planning Commission of Chongqing (General Program) (grant nos. 2011-2-155 and 2012-2-068) and the Scientific and Technological Research Program of Chongqing Municipal Education Commission (grant no. KJ1400223).

\section{References}

1. Neumann E, Schaefer-Ridder M, Wang Y and Hofschneider PH: Gene transfer into mouse lyoma cells by electroporation in high electric fields. EMBO J 1: 841-845, 1982.

2. Yarmush ML, Golberg A, Serša G, Kotnik T and Miklavčič D Electroporation-based technologies for medicine: Principles, applications, and challenges. Annu Rev Biomed Eng 16: 295-320, 2014.

3. Golberg A and Yarmush ML: Nonthermal irreversible electroporation: Fundamentals, applications, and challenges. IEEE Trans Biomed Eng 60: 707-714, 2013.

4. Mir LM, Belehradek M, Domenge C, Orlowski S, Poddevin B, Belehradek J Jr, Schwaab G, Luboinski B and Paoletti C: Electrochemotherapy, a new antitumor treatment: First clinical trial. C R Acad Sci III 313: 613-618, 1991 (In French).

5. Daud AI, DeConti RC, Andrews S, Urbas P, Riker AI, Sondak VK, Munster PN, Sullivan DM, Ugen KE, Messina JL and Heller R: Phase I trial of interleukin-12 plasmid electroporation in patients with metastatic melanoma. J Clin Oncol 26: 5896-5903, 2008. 
6. Granot Y and Rubinsky B: Mass transfer model for drug delivery in tissue cells with reversible electroporation. Int J Heat Mass Transf 51: 5610-5616, 2008.

7. Otten G, Schaefer M, Doe B, Liu H, Srivastava I, zur Megede J, O'Hagan D, Donnelly J, Widera G, Rabussay D, et al: Enhancement of DNA vaccine potency in rhesus macaques by electroporation. Vaccine 22: 2489-2493, 2004.

8. Davalos RV, Mir IL and Rubinsky B: Tissue ablation with irreversible electroporation. Ann Biomed Eng 33: 223-231, 2005

9. Pech M, Janitzky A, Wendler JJ, Strang C, Blaschke S, Dudeck O, Ricke J and Liehr UB: Irreversible electroporation of renal cell carcinoma: A first-in-man phase I clinical study. Cardiovasc Intervent Radiol 34: 132-138, 2011.

10. Cheung W, Kavnoudias H, Roberts S, Szkandera B, Kemp W and Thomson KR: Irreversible electroporation for unresectable hepatocellular carcinoma: Initial experience and review of safety and outcomes. Technol Cancer Res Treat 12: 233-241, 2013.

11. Martin RC II, McFarland K, Ellis S and Velanovich V: Irreversible electroporation therapy in the management of locally advanced pancreatic adenocarcinoma. J Am Coll Surg 215: 361-369, 2012.

12. Martin RC II, McFarland K, Ellis S and Velanovich V: Irreversible electroporation in locally advanced pancreatic cancer: Potential improved overall survival. Ann Surg Oncol 20 (Suppl 3): S443-S449, 2013.

13. Neal RE II, Millar JL, Kavnoudias H, Royce P, Rosenfeldt F, Pham A, Smith R, Davalos RV and Thomson KR: In vivo characterization and numerical simulation of prostate properties for non-thermal irreversible electroporation ablation. Prostate 74 : 458-468, 2014

14. Golberg A, Bruinsma BG, Uygun BE and Yarmush ML: Tissue heterogeneity in structure and conductivity contribute to cell survival during irreversible electroporation ablation by 'electric field sinks'. Sci Rep 5: 8485, 2015.

15. Ellis TL, Garcia PA, Rossmeisl JH Jr, Henao-Guerrero N, Robertson J and Davalos RV: Nonthermal irreversible electroporation for intracranial surgical applications. Laboratory investigation. J Neurosurg 114: 681-688, 2011.

16. Guo Y, Zhang Y, Klein R, Nijm GM, Sahakian AV, Omary RA, Yang GY and Larson AC: Irreversible electroporation therapy in the liver: Longitudinal efficacy studies in a rat model of hepatocellular carcinoma. Cancer Res 70: 1555-1563, 2010.

17. Joyce TA, Wong J, Mittra A, Carpenter S, Haddad D, Carson J, Jayaraman S, Monette S, Solomon SB, Ezell P and Fong Y: Irreversible electroporation is a surgical ablation technique that enhances gene transfer. Surgery 150: 474-479, 2011.
18. Chen L, Wu YY, Liu P, Wang J, Wang G, Qin J, Zhou J and Zhu J: Down-regulation of HPV18 E6, E7, or VEGF expression attenuates malignant biological behavior of human cervical cancer cells. Med Oncol 28 (Suppl 1): S528-S539, 2011.

19. Zhou W, Xiong Z, Liu Y, Yao C and Li C: Low voltage irreversible electroporation induced apoptosis in HeLa cells. J Cancer Res Ther 8: 80-85, 2012.

20. Livak KJ and Schmittgen TD: Analysis of relative gene expression data using real-time quantitative PCR and the 2(-Delta Delta C(T)) method. Methods 25: 402-408, 2001.

21. Rosty C, Sheffer M, Tsafrir D, Stransky N, Tsafrir I, Peter M, de Crémoux P, de La Rochefordière A, Salmon R, Dorval T, et al: Identification of a proliferation gene cluster associated with HPV E6/E7 expression level and viral DNA load in invasive cervical carcinoma. Oncogene 24: 7094-7104, 2005.

22. Doorbar J: Molecular biology of human papillomavirus infection and cervical cancer. Clin Sci (Lond) 110: 525-541, 2006.

23. Breton M and Mir LM: Microsecond and nanosecond electric pulses in cancer treatments. Bioelectromagnetics 33: 106-123, 2012.

24. Jiang C, Davalos RV and Bischof JC: A review of basic to clinical studies of irreversible electroporation therapy. IEEE Trans Biomed Eng 62: 4-20, 2015.

25. Beveridge JR, Wall K, MacGregor SJ, Anderson JG and Rowan NJ: Pulsed electric field inactivation of spoilage microorganisms in alcoholic beverages and the influence of pulse profile. Proc IEEE 92: 1138-1143, 2004.

26. Joyce TA, Mittra A, Song TJ, Cavnar M, Jun K, Carson J, Gholami S, Haddad D, Gaujoux S, Monette S, et al: Irreversible electroporation facilitates gene transfer of a GM-CSF plasmid with a local and systemic response. Surgery 154: 496-503, 2013.

27. Rubinsky B, Onik G and Mikus P: Irreversible electroporation: A new ablation modality-clinical implications. Technol Cancer Res Treat 6: 37-48, 2007. 